\title{
Case report: change of dominant strain during dual SARS-CoV-2 infection
}

\author{
Andrei E. Samoilov ${ }^{*+}$ (D), Valeriia V. Kaptelova ${ }^{*+}$, Anna Y. Bukharina, Olga Y. Shipulina, Elena V. Korneenko, \\ Stepan S. Saenko, Alexander V. Lukyanov, Antonina A. Grishaeva, Antonina A. Ploskireva, \\ Anna S. Speranskaya ${ }^{* \dagger}$ and Vasiliy G. Akimkin ${ }^{\dagger}$
}

\begin{abstract}
Background: The dual infection with SARS-CoV-2 is poorly described and is currently under discussion. We present a study of two strains of SARS-CoV-2 detected in the same patient during the same disease presentation.

Case presentation: A patient in their $90 \mathrm{~s}$ was hospitalised with fever. Oropharyngeal swab obtained on the next day (sample 1) tested positive for SARS-CoV-2. Five days later, the patient was transferred to the ICU (intensive care unit) of the hospital specialising in the treatment of COVID-19 patients, where the patient's condition progressively worsened and continuous oxygen insufflation was required. Repeated oropharyngeal swab (sample 2), which was taken eight days after the first one, also tested positive for SARS-CoV-2. After 5 days of ICU treatment, the patient died. The cause of death was a coronavirus infection, which progressed unfavourably due to premorbid status. We have performed sequencing of full SARS-CoV-2 genomes from oropharyngeal swabs obtained eight days apart. Genomic analysis revealed the presence of two genetically distant SARS-CoV-2 strains in both swabs. Detected strains belong to different phylogenetic clades (GH and GR) and differ in seven nucleotide positions. The relative abundance of strains was 70\% (GH) and 30\% (GR) in the first swab, and 3\% (GH) and 97\% (GR) in the second swab.
\end{abstract}

Conclusions: Our findings suggest that the patient was infected by two genetically distinct SARS-CoV-2 strains at the same time. One of the possible explanations is that the second infection was hospital-acquired. Change of the dominant strain ratio during disease manifestation could be explained by the advantage or higher virulence of the GR clade strain.

Keywords: SARS-CoV-2, COVID-19, Dual infection, High-throughput sequencing, Case report

\section{Background}

Dual infection is a phenomenon where an individual is simultaneously infected with two or more strains of the same virus. It can affect host immune responses and

\footnotetext{
*Correspondence: andrei.samoilov@gmail.com; valeriia.kaptelova@gmail. com; hanna.s.939@gmail.com

${ }^{\dagger}$ Andrei E. Samoilov, Valeriia V. Kaptelova and Anna S. Speranskaya contributed equally as co-first authors

${ }^{\dagger}$ Anna S. Speranskaya and Vasiliy G. Akimkin contributed equally as co-last authors

Central Research Institute of Epidemiology of the Federal Service

for Surveillance on Consumer Rights Protection and Human Wellbeing, 111123 Moscow, Russia
}

result in increased fitness of the viral population. In recent years, a number of cases when individuals were infected with more than one strain of HIV have been identified [1-3]. The findings of dual infections have been reported for influenza viruses [4], the Epstein-Barr virus [5] and other viruses. Cases of SARS-CoV-2 reinfection are reported in the scientific literature [6-8]. Coinfection with respiratory pathogens [9-13], and other viruses, including (but not limited to) HIV (human immunodeficiency virus) [14, 15], Epstein-Barr virus [16], as well as bacterial and fungal confections $[17,18]$ in COVID-19 (coronavirus disease 2019) patients were also described, 
however, there are little to no reports of double infection with SARS-CoV-2 (severe acute respiratory syndrome coronavirus 2), except for in two works $[19,20]$. Here, we present a case report of an individual with two genetically distinct SARS-CoV-2 strains during the same disease manifestation. These strains belonged to different phylogenetic clades: GH and GR. Our findings suggest that the relative abundance of the strains could change significantly over time.

\section{Case presentation}

A patient in their $90 \mathrm{~s}$ with a history of chronic persistent atrial fibrillation, chronic heart failure and hypertension was hospitalised with fever $\left(38{ }^{\circ} \mathrm{C}\right)$, with the admission diagnosis of lobar pneumonia, unspecified organism (J18.1). The patient denied travel or contact with persons with symptoms of COVID-19. Oropharyngeal swab obtained on the next day (sample 1) tested positive for SARS-CoV-2 (cycle threshold, $\mathrm{Ct}=13$, measured using AmpliSens ${ }^{\circledR}$ Cov-Bat-FL assay kit). Five days later, the patient was transferred to the ICU (intensive care unit) of the hospital specialising in the treatment of COVID-19 patients and prescribed Kaletra, Levofloxacin, Clexane, $\mathrm{ACC}$ and Aspirin. During the observation period, the patient's condition progressively worsened and continuous oxygen insufflation was required. Oxygen saturation ranged from $70 \%$ without oxygen support to $92-98 \%$ with oxygen support via a face mask $(5 \mathrm{~L} / \mathrm{min})$. Repeated oropharyngeal swab (sample 2), which was taken eight days after the first one, also tested positive for SARS$\mathrm{CoV}-2$ with high viral load $(\mathrm{Ct}=13$, measured using AmpliSens ${ }^{\circledR}$ Cov-Bat-FL assay kit). After five days of ICU treatment, the patient died. The cause of death was a coronavirus infection, which progressed unfavourably due to premorbid status.

This research was approved by the local ethics committee of the Central Research Institute of Epidemiology of the Federal Service for Surveillance on Consumer Rights Protection and Human Wellbeing on 17.11.2020. Written informed consent was obtained from the patient for publication of this case report and any accompanying images. A copy of the written consent is available for review by the Editor-in-Chief of this journal.

We have performed the sequencing and bioinformatics analysis of two swab samples obtained from the same patient eight days apart using the SCV-2000 bp protocol and Illumina sequencing (see Additional file 1 "Methods" for more detailed description). Briefly, the sequencing of sample 1 yielded $1.1 \mathrm{M}$ paired-end raw reads. After quality filtration and PCR primer trimming, 837 thousand reads remained, $99.87 \%$ of which were mapped to the reference SARS-CoV-2 genome strain hCoV-19/Wuhan/WIV04/2019 (MN996528.1). The mapping of trimmed reads to the reference sequence revealed seven heterogeneous positions (see Additional file 2: Fig. S1 for an illustration).

The sequencing of sample 2 yielded $3.9 \mathrm{M}$ pairedend raw reads. After quality filtration and PCR primer trimming, 3.7 $\mathrm{M}$ reads remained, $99.93 \%$ of which were mapped to the reference SARS-CoV-2 genome strain hCoV-19/Wuhan/WIV04/2019 (MN996528.1). We analysed the mapped reads and found the same heterogeneity at the same positions as in Sample 1, but at a much lower level.

We interpreted our observations as the simultaneous presence of two SARS-CoV-2 strains in the same patient's samples. After obtaining consensus genomic sequence from the dominant strain from the less heterogeneous second sample (hereafter referred to as strain 2 ), it became possible to unambiguously reconstruct genomic sequences of the strain prevalent in the first sample (hereafter referred to as strain 1). The relative abundance of strains 1 and 2 in both time points was assessed by averaging the relative coverage of heterogeneous positions (Fig. 1A) and amounted to roughly $69 \%$ and $31 \%$ in the first sample and 3\% and $97 \%$ in the second sample, respectively (Fig. 1C). We found that strain 1 was dominant in sample 1 , and strain 2 became dominant in sample 2.

The resulting sequences are available at GenBank with accession numbers MW305251.1 (dominant strain from Sample 1) and MW305250.1 (the dominant strain from Sample 2, collected eight days after the first swab).

Heterogeneity in the sequence reads can also be explained by sequencing artifacts arising from the polymerase errors, chimeric fragments generation during nucleic acid amplification, and contamination during RNA extraction and library preparation. To exclude the possibility that the observed heterogeneity is a mistake, we isolated RNA from the original swabs for the second time and performed sequencing of libraries prepared from total RNA without any enrichment for both samples.

RNA-seq of sample 1 yielded $12.6 \mathrm{M}$ paired-end $250 \mathrm{bp}$ long reads. After quality filtration and PCR primer trimming, $12.2 \mathrm{M}$ reads remained, $2.29 \%$ of which were mapped to the reference SARS-CoV-2 genome strain hCoV-19/Wuhan/WIV04/2019 (MN996528.1). We compared RNA-seq reads with reads obtained using the SCV-2000 bp protocol (Fig. 1B). Roughly the same frequencies of alternative nucleotides prove that observed heterogeneity was not a result of a sequencing artifact and that DNA originating from two different SARS-CoV-2 strains is present in sample 1. Read coverage at the genomic positions 

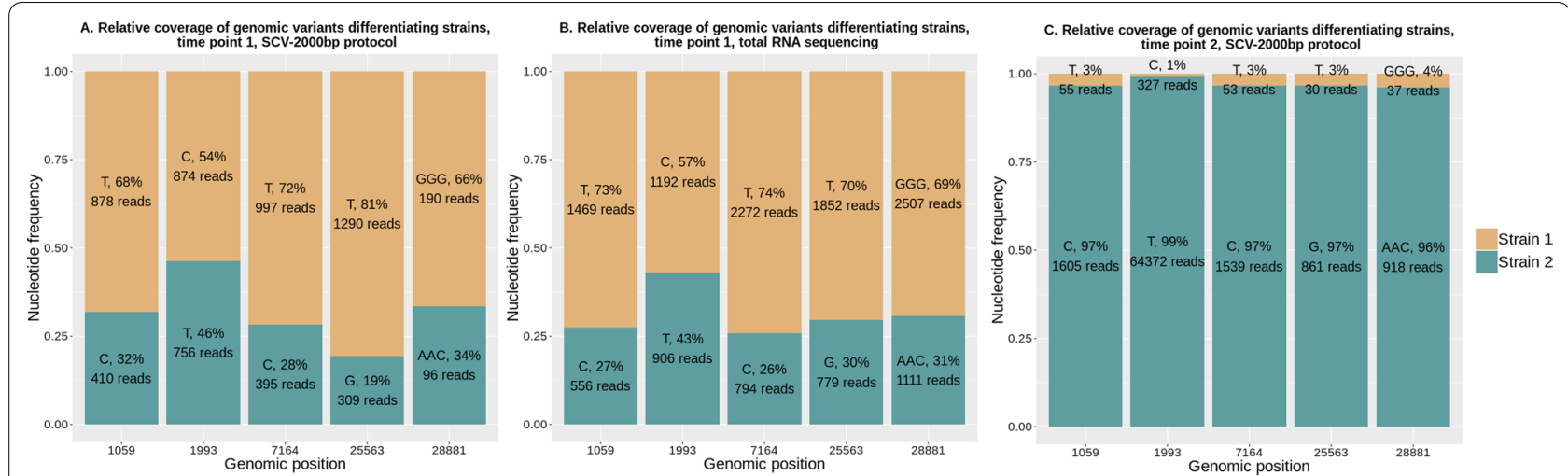

Fig. 1 Relative coverage of SARS-CoV-2 genomic variants, which differentiate strains 1 and 2. Genomic positions are at the X-axis, their relative frequencies are at Y-axis. A Sample 1, sequencing using genome fragments amplification (SCV-2000 bp protocol); B Sample 1, sequencing using total RNA library; C Sample 2 (collected eight days later), sequencing using genome fragments amplification (SCV-2000 bp protocol)

differentiating strain 1 from strain 2 varied from 2100 to 4090 in sample 1 after total RNA sequencing.

RNA-seq of Sample 2 yielded 15.0 M paired-end raw reads. After quality filtration and PCR primer trimming, $14.6 \mathrm{M}$ reads remained, and only $0.04 \%$ of them were mapped to the reference SARS-CoV-2 genome strain hCoV-19/Wuhan/WIV04/2019 (MN996528.1), which was not enough to confirm the presence of a minor fraction of reads (about 3\%) representing strain 1.

To confirm the presence of two different strains in the same sample, we have designed a set of 16 primers pairs (Additional file 3) aimed at the position of GGG28881AAC mutation (present in GR clade and absent in others, including $\mathrm{GH}$ ). One of the primers from the pair overlaps one or two nucleotides at the positions $28,881-28,883$ (either GGG or AAC) at the $3^{\prime}$ end, so every primer pair is expected to uniquely amplify the fragment of GR clade genome (8 primer pairs) or non-GR clade genome (8 primer pairs). We have performed PCR with all of the designed primers pairs with the cDNA from both samples, successfully obtained amplicons, and performed high-throughput sequencing. We have mapped paired reads to the reference sequence and discovered the GGG28881AAC mutation in the amplicons aimed at GR clade or absence of this mutation in the amplicons aimed at non-GR clade for both sample 1 and sample 2 with nearly $100 \%$ frequency for the most of the primer pairs (see Additional file 4: Fig. S2 for an illustration). This experiment confirms the presence of two different strains belonging to different GISAID clades in both samples.

Paired sequence reads were deposited to SRA with the accession number PRJNA719737 in the NCBI BioProject database, SAMN18616569 and SAMN18616570 stand for sample 1 and sample 2, respectively.
Comparison of strain 1 genomic sequence to all of the GISAID SARS-CoV-2 database (as of 11 November 2020) revealed that this sequence is unique to GISAID, the closest genomes having at least two mismatches compared to strain 1 genome. Out of 571 closest sequences, only three originated from Russia (EPI_ISL_428905, EPI ISL_428875 and EPI_ISL_428871, all of them were collected in March), most of the other genomes originated from the USA (402), Iceland (28) and Canada (26) and were collected in March-early April.

Comparison of strain 2 genomic sequence to all of the GISAID SARS-CoV-2 database (as of 11 November 2020) revealed 1062 genomic sequences with $100 \%$ identity to strain 2, 78 out of which originated from different regions of Russia, with 10 of them collected in Moscow (collection dates of which vary from late March to early April), including eight genomes obtained in our lab, as described in [21]. The latest genomes with $100 \%$ identity to strain 2 were collected in Saint-Petersburg in the middle of September (EPI_ISL_602339 and EPI_ISL_602340). Other genomes with $100 \%$ identity to strain 2 originated mostly from England (333), Portugal (121) and the USA (86) and were collected mainly in March and April.

Comparison of strain 1 and strain 2 genomic sequences with the reference strain hCoV-19/Wuhan/WIV04/2019 (MN996528.1) revealed four nucleotide mutations present in both of them (C241T in the non-coding region; C3037T, a synonymous substitution in NSP3 protein; C14408T, resulting in P323L mutation in NSP12 protein; and A23403G, resulting in D614G mutation in spike protein), as well as four mutations present only in strain 1 (C1059T, resulting in T85I mutation in NSP2 protein; T1993C, a synonymous substitution in NSP2 protein; C7164T, resulting in T1482I mutation in NSP3 protein; and G25563T, resulting in Q57H in NS3 protein) and 
three mutations present only in strain 2 (G28881A and G28882A, resulting in $\mathrm{R} 203 \mathrm{~K}$ mutation in $\mathrm{N}$ protein; G28881C, resulting in G204R mutation in $\mathrm{N}$ protein) (Table 1).

Phylogenetic analysis was performed by building a tree of all of the available SARS-CoV-2 genomes from the samples collected in Russia. It has been revealed that strain 1 (MW305251.1) belongs to the GH clade, and strain 2 (MW305250.1) belongs to the GR clade (GISAID classification) (see Fig. 2).

\section{Discussion and conclusions}

Dual infection can affect host immune responses and result in increased fitness of the viral population. HIV dual infection contributes to rapid disease progression [1] and increased viral load [2], and requires antiretroviral treatment effective against both viruses [3]. Meanwhile, despite a rapidly growing body of evidence, there is little to no information about dual SARS-CoV-2 infection. To our knowledge, the possibility of SARS-CoV-2 double infection was discussed in two works $[19,20]$. Authors of both provided no information about patients' medical history and viral subpopulation dynamics during disease progression, and almost no information about clade classification of SARS-CoV-2 analysed strains.

Liu et al. [19] cite the CEO of CODE Genetics biopharmaceutical company Kari Stefansson, who has reported a patient hospitalized in Iceland infected by two SARS-CoV-2 subtypes simultaneously in early March 2020. One strain of the SARS-CoV-2 coronavirus was more aggressive, while the second strain is a mutation from the original version of the coronavirus that appeared in Wuhan, China. This was regarded

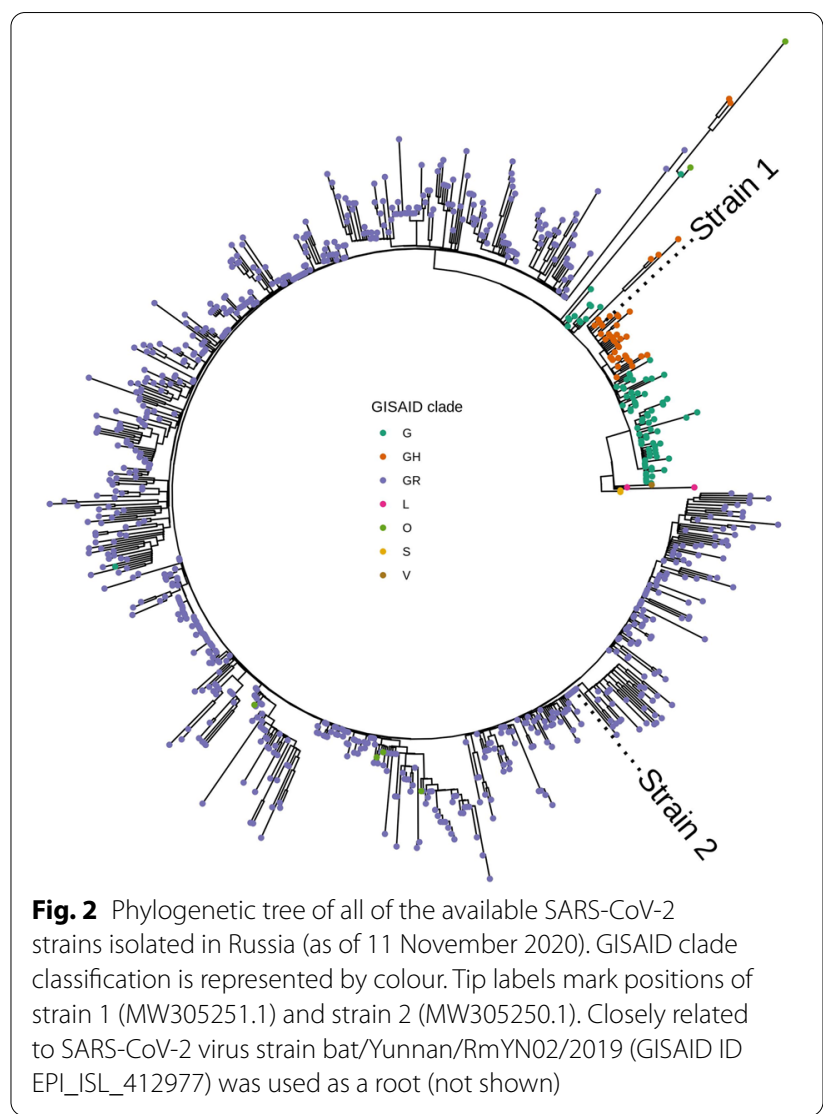

as the first known case of co-infection. Authors have detected the presence of signature mutations from different phylogenetic groups in the same genomes and explained it as possible co-infection or homogenous

Table 1 List of mutations present in strains 1 and 2

\begin{tabular}{|c|c|c|c|c|c|c|c|c|c|}
\hline \multicolumn{4}{|c|}{ Reference (MN996528.1) } & \multicolumn{3}{|c|}{ Strain 1 (MW305251.1) } & \multicolumn{3}{|c|}{ Strain 2 (MW305250.1) } \\
\hline Position & Nucleotide & Protein & Amino acid & Nucleotide & Amino acid & Mutation name & Nucleotide & Amino acid & Mutation name \\
\hline 241 & C & - & - & $\mathrm{T}$ & - & - & $\mathrm{T}$ & - & - \\
\hline 1059 & C & NSP2 & $\mathrm{T}$ & $\mathbf{T}$ & $\mathrm{I}$ & T85I & C & $\mathrm{T}$ & - \\
\hline 1993 & $\mathrm{~T}$ & NSP2 & Y & $\mathrm{C}$ & $\mathrm{Y}$ & $\begin{array}{l}\text { Synonymous sub- } \\
\text { stitution }\end{array}$ & $\mathrm{T}$ & Y & $\begin{array}{l}\text { Synonymous substi- } \\
\text { tution }\end{array}$ \\
\hline 3037 & C & NSP3 & $\mathrm{F}$ & $\mathrm{T}$ & $\mathrm{F}$ & $\begin{array}{l}\text { Synonymous substi- } \\
\text { tution }\end{array}$ & $\mathrm{T}$ & $\mathrm{F}$ & $\begin{array}{l}\text { Synonymous substi- } \\
\text { tution }\end{array}$ \\
\hline 7164 & C & NSP3 & $\mathrm{T}$ & $\mathbf{T}$ & 1 & T1482I & C & $\mathrm{T}$ & - \\
\hline 14,408 & C & NSP12 & $P$ & $\mathrm{~T}$ & $\mathrm{~L}$ & P323L & $\mathrm{T}$ & $\mathrm{L}$ & P323L \\
\hline 23,403 & A & Spike & $\mathrm{D}$ & G & G & D614G & G & G & D614G \\
\hline 25,563 & G & NS3 & Q & $\mathbf{T}$ & $\mathrm{H}$ & Q57H & G & - & - \\
\hline 28,881 & G & $N$ & $\mathrm{R}$ & G & $\mathrm{R}$ & - & A & $\mathrm{K}$ & R203K \\
\hline 28,882 & G & $\mathrm{N}$ & $\mathrm{R}$ & G & $\mathrm{R}$ & - & A & $\mathrm{K}$ & \\
\hline 28,883 & G & $\mathrm{N}$ & G & G & G & - & $C$ & $\mathbf{R}$ & G204R \\
\hline
\end{tabular}


recombination. However, it can also be explained as contamination or sequencing errors, and reliable confirmation of the authors' hypothesis requires a more indepth analysis of obtained data.

Hashim et al. [20] utilized Sanger sequencing to obtain short (795 bp) fragments of spike protein gene. They have discovered double peaks and interpreted it as double infection in all 19 analysed samples, with most of the detected mutations being missense. The authors discussed that co-infecting strains could compensate for the damaging effect of the truncated spike protein. Our observations show different dynamics between two strains, where one was replaced with another after several days. In our opinion, the results of Hashim et al. need to be confirmed using high-throughput sequencing technology because the authors' interpretation of Sanger sequencing data is questionable. Most of the electropherograms presented in the manuscript [20] consist of double peaks with low intensity of the minor peak, which makes interpretation of the obtained data challenging. Minor variants can be reliably detected in the $20 \%$ mixture analysed trace in conventional Sanger sequencing; however, in the analysed traces from the $10 \%$ and $5 \%$ mixtures, the fragment is indistinguishable from the baseline noise [22].

The mutation rate of SARS-CoV-2 inside the same host is a critical parameter for understanding viral evolution because it may become more infectious or more virulent. Choi et al. [23] described the case of persistent infection (over 150 days) accompanied by accelerated evolution of SARS-CoV-2 in an immunocompromised patient. On days 18 and 25, sequencing of SARS-CoV-2 genomes obtained from the patient revealed five amino acid substitutions compared to the reference, but later their number grew to over 20. The largest number of mutations was detected in the Spike protein, especially in the receptorbinding domain. We have not observed the appearance of new mutations, but strain 1, discussed in this work, is closely related to the strain obtained from the immunocompromised patient on day 18 (both of them possess mutations Spike_D614G, NS3_Q57H, NSP2_T85I and NSP12_P323L, but differ in mutation NSP13_T115I present only in the strain obtained from the immunocompromised patient and mutation NSP3_T1482I present only in strain 1).

SARS-CoV-2 quasispecies and genetic diversity within the same individual or cell culture were discussed in several articles and preprints [24-28]. Low fidelity of RNA polymerases results in heterogeneity in the RNA virus population. Here, we report a dual infection because two strains we detected belong to different phylogenetic clades, which cannot be explained by RNA polymerase errors.
The presence of two viral variants in the same patient might be associated with nosocomial infection. However, the patient spent only one day in the hospital before the first swab was collected, in which we have detected two SARS-CoV-2 strains. The probability of getting a positive PCR test result in the early days after infection is rather low [29]. Therefore, our data can be interpreted either as an infection with two strains before admission to the hospital or as a rapid increase in hospital strain viral load to a detectable level due to the patient's weakened immune system. The severity and rapid progression of the disease, along with unchangeably high viral load $(\mathrm{Ct}=13)$, could be associated with either a change in the dominant strain of SARS-CoV-2 or the patient's elderly age [30].

Our results show a drastic change in both strains' abundance: the dominant strain from the first sample almost disappeared in the sample obtained a week later. The strain dominating in the first sample belongs to the GH clade, while the strain which prevailed in the second sample belongs to the GR clade. Change of the dominant strain in the viral community can be a stochastic event, or it can be caused by many factors, including exposure to nosocomial infection, the individuality of the host immune response, or difference in strain fitness. Our experiment's design does not allow us to find out the cause of change in the viral community (we have analyzed only two samples taken from a single patient), but it allows us to detect mutations differentiating one strain from another and discuss their potential effect on strain fitness. A longitudinal study of similar cases with double infection can potentially shed light on the connection between mutations in the SARS-CoV-2 genome and change of strain abundance in the viral community.

In our case, potentially disadvantageous mutations (present in strain 1, which decreased its abundance over time) include Q57H in NS3 protein, T85I in NSP2 protein and T1482I in NSP3 protein. NS3_Q57H demarcates the GH clade [31]. As of 11 November 2020 , it occurs in $4.6 \%$ ( 40 out of 874 ) of Russian SARS$\mathrm{CoV}-2$ sequences. Possible effects of this mutation were discussed in several papers. In a work by Gupta et al. [32], who used protein modelling, its effect was predicted as deleterious. Alam et al. discussed that it prevents ion permeability by constricting the channel pore more tightly, possibly reducing viral release and immune response [33]. In work by Wang et al. [34], the authors suggested that it can make the SARS-CoV-2 more infectious. The second mutation present only in strain 1, NSP2_T85I, is rare in Russia and occurs in 3.4\% (30 out of 874) Russian SARS-CoV-2 genomes (as of 11 November 2020). This mutation also has predicted deleterious functional outcome [35]. Wang et al. [34] discussed that this mutation benefits from other 
mutations like Spike_D614G and NS3_Q57H and could enhance infectivity. Finally, NSP3_T1482I mutation is present in only 96 genomes submitted to GISAID (as of 11 November 2020), and, to our knowledge, it was never discussed in scientific literature.

Potentially advantageous mutations (present in the dominant strain in the second sample) include R203K and G204R in N protein. These mutations occur together as a result of the substitution of three consecutive nucleotides. The presence of these mutations demarcates the GR clade [31]. Over $85 \%$ of Russian SARS-CoV-2 genomes submitted to GISAID (as of 11 November 2020) belong to the GR clade and possess both of these mutations. According to different protein modelling approaches, these mutations either destabilise $\mathrm{N}$ protein [33] or have a neutral effect [32]. Several articles point out the association of this clade with higher mortality [36] or significant prevalence in severely ill or deceased patients and also higher prevalence in females and children compared to other clades [37].

Other mutations present in both strains are D614G in spike protein and P323L in NSP12. Spike_D614G is one of the most widely discussed mutations. Its presence demarcates the G clade [31], it increases infectivity [3840] and mortality [41, 42], alters viral fitness [43], and, according to protein modelling, enhances the folding stability of the spike protein [34]. It is present in $99.2 \%$ of SARS-CoV-2 genomes obtained from Russia (as of 11 November 2020). NSP12_P323L is predicted to make the polymerase more rigid, which may increase the replication speed [34] and mutation rate [44, 45]. It is present in 97.9\% of Russian SARS-CoV-2 genomes (as of 11 November 2020).

In our work, we present a case of dual SARS-CoV-2 infection, which allowed us to discuss the potential difference in the relative fitness of two genetically distant strains. The effect of SARS-CoV-2 dual infection on viral load and the severity or duration of COVID-19 is currently unknown. It is possible that the presence of two different SARS-CoV-2 strains was a factor which led to rapid progression of the patient's disease and death. The importance of research aimed at cases similar to ours can hardly be overestimated because it provides insights into the molecular epidemiology of COVID-19 and can help detect potentially advantageous mutations which increase virulence and fitness of SARS-CoV-2.

Our study shows the case of dual SARS-CoV-2 infection by two phylogenetically distant strains and the viral community dynamics. Dominant strain from the first sample belonged to GH clade, while GR clade strain became dominant eight days after collecting the first sample.

\section{Abbreviations}

CDNA: Complementary deoxyribonucleic acid; COVID-19: Coronavirus disease 2019; Ct: Cycle threshold; HIV: Human immunodeficiency virus; ICU: Intensive care unit; PCR: Polymerase chain reaction; RNA: Ribonucleic acid; SARS-CoV-2: Severe acute respiratory syndrome coronavirus 2 .

\section{Supplementary Information}

The online version contains supplementary material available at https://doi. org/10.1186/s12879-021-06664-w.

Additional file 1. Description of methods used for SARS-CoV-2 full genome sequencing and data analysis.

Additional file 2: Figure S1. IGV snapshot showing the heterogeneity in mapped Illumina reads in the first sample. $66 \%$ of the reads match the reference SARS-CoV-2 sequence strain hCoV-19/Wuhan/WIV04/2019 (MN996528.1), 34\% of reads have GGG->AAC substitution at positions $28,881-28,883$.

Additional file 3. List of primer pairs used to confirm the presence of two viral strains from different clades in the same sample. One of the primers from the pair overlaps one or two nucleotides at the positions 28881-28883 (either GGG or AAC) at the $3^{\prime}$ end, so every primer pair is expected to uniquely amplify the fragment of $G R$ clade genome with GGG28881AAC mutation (8 primer pairs with GR in the name of one of the primers) or non-GR clade genome (8 primer pairs with $\mathrm{GH}$ in the name of one of the primers).

Additional file 4: Figure S2. IGV snapshot showing the result of the sequencing of PCR products obtained using primers uniquely amplifying a fragment of viral genome with (two bottom libraries, GR clade) or without (two upper libraries, non-GR clade) GGG28881AAC mutation. All of the PCR products shown were amplified using CDNA from sample 2.

\section{Acknowledgements}

The authors would like to express their gratitude to the staff of the Molecular diagnostic laboratory of Central Research Institute of Epidemiology of the Federal Service for Surveillance on Consumer Rights Protection and Human Wellbeing for providing the anonymised samples of patients with COVID-19.

\section{Authors' contributions}

Conceptualization, A.S.S., A.E.S. and V.V.K.; methodology, A.S.S. and V.V.K.; validation, A.S.S., V.V.K., A.E.S. and A.Y.B.; formal analysis, A.E.S.; investigation, A.S.S., V.V.K., S.S.S. and A.Y.B.; resources, O.Y.S., A.V.L., A.A.G., A.A.P. and V.G.A.; data curation, O.Y.S., A.V.L., A.A.G., A.A.P., V.V.K., E.V.K., V.G.A. and A.E.S.; writingoriginal draft preparation, A.S.S., A.E.S. and V.V.K.; writing — review and editing, A.S.S., A.E.S., V.V.K. and E.V.K.; visualization, A.E.S.; supervision, A.S.S. and V.G.A.; project administration, V.G.A.; funding acquisition, A.S.S. All authors have read and approved the final manuscript.

\section{Funding}

This work was supported by the Ministry of Science and Higher Education of the Russian Federation within the framework of a grant in the form of a subsidy for the creation and development of the 'World-class Genomic Research Centre for Ensuring Biological Safety and Technological Independence under the Federal Scientific and Technical Program for the Development of Genetic Technologies', agreement No. 075-15-2019-1666. The funder had no role in study design, data collection and analysis, decision to publish, or preparation of the manuscript.

\section{Availability of data and materials}

Full-genome sequences of strain 1 and 2 are available at GenBank using accession numbers MW305251.1 and MW305250.1, respectively. Paired sequence reads were deposited to SRA with the accession number PRJNA719737 in the NCBI BioProject database. 


\section{Declarations}

\section{Ethics approval and consent to participate}

This research was approved by the local ethics committee of the Central Research Institute of Epidemiology of the Federal Service for Surveillance on Consumer Rights Protection and Human Wellbeing on 17.11.2020.

\section{Consent for publication}

Written informed consent for publication of their details was obtained from the patient.

\section{Competing interests}

The authors declare that they have no competing interests.

\section{Received: 23 December 2020 Accepted: 7 September 2021}

Published online: 16 September 2021

\section{References}

1. Gottlieb GS, Nickle DC, Jensen MA, Wong KG, Grobler J, Li F, et al. Dual HIV-1 infection associated with rapid disease progression. Lancet. 2004;363:619-22

2. van der Kuyl AC, Cornelissen M. Identifying HIV-1 dual infections. Retrovirology. 2007;4:67.

3. Ekouevi DK, Eholie SP. Update on HIV-1 and HIV-2 dual infection. In: Encyclopedia of AIDS. Springer New York: New York, NY, 2013, pp 1-10.

4. Myers CA, Kasper MR, Yasuda CY, Savuth C, Spiro DJ, Halpin R, et al. Dual infection of novel influenza viruses $\mathrm{A} / \mathrm{H} 1 \mathrm{~N} 1$ and $\mathrm{A} / \mathrm{H} 3 \mathrm{~N} 2$ in a cluster of Cambodian patients. Am J Trop Med Hyg. 2011;85:961-3.

5. Weinberg A, Bloch KC, Li S, Tang Y, Palmer M, Tyler KL. Dual infections of the central nervous system with Epstein-Barr virus. J Infect Dis. 2005:191:234-7.

6. Tillett RL, Sevinsky JR, Hartley PD, Kerwin H, Crawford N, Gorzalski A, et al. Genomic evidence for reinfection with SARS-CoV-2: a case study. Lancet Infect Dis. 2020;21:52-8. https://doi.org/10.1016/S1473-3099(20)30764-7.

7. Tomassini S, Kotecha D, Bird PW, Folwell A, Biju S, Tang JW. Setting the criteria for SARS-CoV-2 reinfection — six possible cases. J Infect. 2020;82:282327. https://doi.org/10.1016/j.jinf.2020.08.011.

8. Van Elslande J, Vermeersch P, Vandervoort K, Wawina-Bokalanga T, Vanmechelen $B$, Wollants $E$, et al. Symptomatic SARS-CoV-2 reinfection by a phylogenetically distinct strain. Clin Infect Dis. 2020. https://doi.org/10. 1093/cid/ciaa1330.

9. Wang W, Le Grange JM, Wang X, Du S, Li C, et al. Coinfection of SARS-CoV-2 and other respiratory pathogens. Infect Drug Resist. 2020;13:3045-53.

10. Miatech JL, Tarte NN, Katragadda S, Polman J, Robichaux SB. A case series of coinfection with SARS-CoV-2 and influenza virus in Louisiana. Respir Med Case Rep. 2020;31: 101214.

11. Castillo EM, Coyne CJ, Brennan JJ, Tomaszewski CA. Rates of coinfection with other respiratory pathogens in patients positive for coronavirus disease 2019 (COVID-19). J Am Coll Emerg Physicians Open. 2020;1:592-6.

12. Calcagno A, Ghisetti $V$, Burdino E, Trunfio M, Allice T, Boglione $L$, et al. Coinfection with other respiratory pathogens in COVID-19 patients. Clin Microbiol Infect. 2020;27:297-8. https://doi.org/10.1016/j.cmi.2020.08 012.

13. Sharov KS. SARS-CoV-2-related pneumonia cases in pneumonia picture in Russia in March-May 2020: secondary bacterial pneumonia and viral co-infections. J Glob Health. 2020;10:20504.

14. Kanwugu ON, Adadi P. HIV/SARS-CoV-2 coinfection: a global perspective. J Med Virol. 2020:93:726-32. https://doi.org/10.1002/jmv.26321.

15. Johnston R. The first 6 months of HIV-SARS-CoV-2 coinfection: outcomes for 6947 individuals. Curr Opin HIV AIDS. 2021;16:54-62.

16. Roncati L, Lusenti B, Nasillo V, Manenti A. Fatal SARS-CoV-2 coinfection in course of EBV-associated lymphoproliferative disease. Ann Hematol. 2020;99:1945-6.

17. Chen $X$, Liao $B$, Cheng $L$, Peng $X, X u X$, Li Y, et al. The microbial coinfection in COVID-19. Appl Microbiol Biotechnol. 2020;104:7777-85.

18. Hughes S, Troise O, Donaldson H, Mughal N, Moore LSP. Bacterial and fungal coinfection among hospitalized patients with COVID-19: a retrospective cohort study in a UK secondary-care setting. Clin Microbiol Infect. 2020;26:1395-9.

19. Liu S, Shen J, Fang S, Li K, Liu J, Yang L, et al. Genetic spectrum and distinct evolution patterns of SARS-CoV-2. Front Microbiol. 2020;11:2390. https://doi.org/10.3389/fmicb.2020.593548.

20. Hashim H, Mohammed M, Mousa M, Abdulameer H, Alhassnawi A, Hassan S, et al. Infection with different strains of SARS-COV-2 in patients with COVID-19. Arch Biol Sci. 2020;72(575-585):51-51.

21. Speranskaya AS, Kaptelova W, Valdokhina AV, Bulanenko VP, Samoilov AE, Korneenko EV, et al. SCV-2000bp: a primer panel for SARS-CoV-2 fullgenome sequencing. bioRxiv. 2020. https://doi.org/10.1101/2020.08.04. 234880.

22. Davidson CJ, Zeringer E, Champion KJ, Gauthier M-P, Wang F, Boonyaratanakornkit J, et al. Improving the limit of detection for Sanger sequencing: a comparison of methodologies for KRAS variant detection. Biotechniques. 2012:53:182-8.

23. Choi B, Choudhary MC, Regan J, Sparks JA, Padera RF, Qiu X, et al. Persistence and evolution of SARS-CoV-2 in an Immunocompromised Host. N Engl J Med. 2020;383:2291-3.

24. Kuipers J, Batavia AA, Jablonski KP, Bayer F, Borgsmüller N, Dondi A, et al. Within-patient genetic diversity of SARS-CoV-2. bioRxiv. 2020. https://doi. org/10.1101/2020.10.12.335919.

25. Nyayanit D, Yadav P, Kharde R, Shete-Aich A. Quasispecies analysis of the SARS-CoV-2 from representative clinical samples: a preliminary analysis. Indian J Med Res. 2020;152:105.

26. Jary A, Leducq V, Malet I, Marot S, Klement-Frutos E, Teyssou E, et al. Evolution of viral quasispecies during SARS-CoV-2 infection. Clin Microbiol Infect. 2020;26(1560):e1-1560.e4.

27. Chaudhry MZ, Eschke K, Grashoff M, Abassi L, Kim Y, Brunotte L, et al. SARS-CoV-2 quasispecies mediate rapid virus evolution and adaptation. bioRxiv. 2020. https://doi.org/10.1101/2020.08.10.241414.

28. Zhang Z, Wang F-S. SARS-associated coronavirus quasispecies in individual patients. N Engl J Med. 2004;350:1366-7.

29. Kucirka LM, Lauer SA, Laeyendecker O, Boon D, Lessler J. Variation in false-negative rate of reverse transcriptase polymerase chain reactionbased SARS-CoV-2 tests by time since exposure. Ann Intern Med. 2020;173:262-7.

30. Wang W, Tang J, Wei F. Updated understanding of the outbreak of 2019 novel coronavirus (2019-nCoV) in Wuhan, China. J Med Virol. 2020;92:441-7.

31. Islam OK, Al-Emran HM, Hasan MS, Anwar A, Jahid MIK, Hossain MA Emergence of European and North American mutant variants of SARSCoV-2 in South-East Asia. Transbound Emerg Dis. 2020;68:824-32. https:// doi.org/10.1111/tbed.13748.

32. Gupta AM, Chakrabarti J, Mandal S. Non-synonymous mutations of SARSCoV-2 leads epitope loss and segregates its variants. Microbes Infect. 2020:22:598-607.

33. Alam ASMRU, Islam OK, Hasan S, Al-Emran HM, Jahid IK, Anwar HM. Evolving infection paradox of SARS-CoV-2: fitness costs virulence? medRxiv. 2020. https://doi.org/10.31219/osf.io/y36ve.

34. Wang R, Chen J, Gao K, Hozumi Y, Yin C, Wei G-W. Characterizing SARSCoV-2 mutations in the United States. arXiv preprint arXiv 2020. http:// arxiv.org/abs/2007.12692

35. Chan AP, Choi Y, Schork NJ. Conserved genomic terminals of SARS-CoV-2 as coevolving functional elements and potential therapeutic targets. mSphere. 2020;5: e00754-20.

36. Farkas C, Mella A, Haigh JJ. Large-scale population analysis of SARSCoV-2 whole genome sequences reveals host-mediated viral evolution with emergence of mutations in the viral Spike protein associated with elevated mortality rates. medRxiv. 2020. https://doi.org/10.1101/2020.10. 23.20218511

37. Hamed S, Elkhatib W, Khairallah A, Noreddin A. Global dynamics of SARS-CoV-2 clades and their relation to COVID-19 epidemiology. 2020. doi:https://doi.org/10.21203/rs.3.rs-89876/v1.

38. Zhang L, Jackson CB, Mou H, Ojha A, Peng H, Quinlan BD, et al. SARSCoV-2 spike-protein D614G mutation increases virion spike density and infectivity. Nat Commun. 2020;11:6013.

39. Korber B, Fischer WM, Gnanakaran S, Yoon H, Theiler J, Abfalterer W, et al. Tracking changes in SARS-CoV-2 spike: evidence that D614G increases infectivity of the COVID-19 virus. Cell. 2020;182:812-827.e19. 
40. Nie J, Zhang L, Hao H, Liu S, et al. The impact of mutations in SARS-CoV-2 spike on viral infectivity and antigenicity. Cell. 2020;182:1284-1294.e9.

41. Becerra-Flores M, Cardozo T. SARS-CoV-2 viral spike G614 mutation exhibits higher case fatality rate. Int J Clin Pract. 2020. https://doi.org/10.1111/ ijcp.13525.

42. Eaaswarkhanth M, Al Madhoun A, Al-Mulla F. Could the D614G substitution in the SARS-CoV-2 spike (S) protein be associated with higher COVID-19 mortality? Int J Infect Dis. 2020;96:459-60.

43. Plante JA, Liu Y, Liu J, Xia H, Johnson BA, Lokugamage KG, et al. Spike mutation D614G alters SARS-CoV-2 fitness. Nature. 2020;592:116-21. https://doi.org/10.1038/s41586-020-2895-3.

44. Eskier D, Karakülah G, Suner A, Oktay Y. RdRp mutations are associated with SARS-CoV-2 genome evolution. PeerJ. 2020;8: e9587.
45. Begum F, Mukherjee D, Das S, Thagriki D, Tripathi PP, Banerjee AK, et al. Specific mutations in SARS-CoV2 RNA dependent RNA polymerase and helicase alter protein structure, dynamics and thus function: Effect on viral RNA replication. bioRxiv. 2020. https://doi.org/10.1101/2020.04.26. 063024.

\section{Publisher's Note}

Springer Nature remains neutral with regard to jurisdictional claims in published maps and institutional affiliations.
Ready to submit your research? Choose BMC and benefit from:

- fast, convenient online submission

- thorough peer review by experienced researchers in your field

- rapid publication on acceptance

- support for research data, including large and complex data types

- gold Open Access which fosters wider collaboration and increased citations

- maximum visibility for your research: over $100 \mathrm{M}$ website views per year

At BMC, research is always in progress.

Learn more biomedcentral.com/submissions 